\title{
Development of a Model-Based Clinical Sepsis Biomarker for Critically Ill Patients
}

\author{
Jessica Lin*. Jacquelyn D. Parente**. J. Geoffrey Chase**, Geoffrey M. Shaw*, Amy J. \\ Blakemore**, Aaron J. LeCompte**, Christopher Pretty**, Normy N. Razak**, Dominic \\ S. Lee***, Christopher E. Hann**, Sheng-Hui Wang* \\ *University of Otago Christchurch, Department of Medicine, Christchurch, New Zealand (e-mail: \\ jessica.lin@otago.ac.nz). \\ **University of Canterbury, Mechanical Engineering Department, Centre for Bioengineering, \\ Christchurch, New Zealand \\ *** University of Canterbury, Mathematics and Statistics Department, Christchurch, New Zealand
}

\begin{abstract}
Sepsis occurs frequently in the intensive care unit (ICU) and is a leading cause of admission, mortality, and cost. Treatment guidelines recommend early intervention, however positive blood culture results may take up to 48 hours. Insulin sensitivity $\left(\mathrm{S}_{\mathrm{I}}\right)$ is known to decrease with worsening condition and could thus be used to aid diagnosis. Some glycemic control protocols are able to accurately identify insulin sensitivity in realtime.

Hourly model-based insulin sensitivity $S_{I}$ values were calculated from glycemic control data of 36 patients with sepsis. The hourly $S_{I}$ is compared to the hourly sepsis score (ss) for these patients (ss $=0-4$ for increasing severity). A multivariate clinical biomarker was also developed to maximize the discrimination between different ss groups. Receiver operator characteristic (ROC) curves for severe sepsis (ss $\geq 2$ ) are created for both $S_{I}$ and the multivariate clinical biomarker.

Insulin sensitivity as a sepsis biomarker for diagnosis of severe sepsis achieves a $50 \%$ sensitivity, 76\% specificity, 4.8\% positive predictive value (PPV), and 98.3\% negative predictive value (NPV) at an $\mathrm{S}_{\mathrm{I}}$ cut-off value of $0.00013 \mathrm{~L} / \mathrm{mU} / \mathrm{min}$. Multivariate clinical biomarker combining $S_{\mathrm{I}}$, temperature, heart rate, respiratory rate, blood pressure, and their respective hourly rates of change achieves $73 \%$ sensitivity, $80 \%$ specificity, $8.4 \%$ PPV, and 99.2\% NPV. Thus, the multivariate clinical biomarker provides an effective real-time negative predictive diagnostic for severe sepsis. Examination of both inter- and intra-patient statistical distribution of this biomarker and sepsis score shows potential avenues to improve the positive predictive value.
\end{abstract}

Keywords:

sepsis, insulin sensitivity, biomarker, diagnosis, receiver operator characteristic, glucose control, real-time clinical application. 


\section{INTRODUCTION}

Sepsis presents a serious medical problem in the adult intensive care unit (ICU) around the world, with a $11-15 \%$ incidence of severe sepsis, 30-60\% mortality rate, $\$ 22,100$ USD average cost per case, $\$ 16.7$ billion USD annual total cost, and $1.5 \%$ projected annual incidence increase [1]. Sepsis treatment guidelines and patient management protocols recommend early goal-directed resuscitation of the septic patient during the first 6 hours after infection recognition [2]. Currently, blood bacteria cultures are considered the gold standard for confirmation of infection. However, only $51 \%$ of sepsis cases are positively identified as cultured pathogens [3].

Early interventions have been documented to reduce mortality from $46.5 \%$ to $30.5 \%$ [4]. In addition, a landmark clinical trial implementing a blood glucose control protocol resulted in a reduction in the incidence of sepsis [5]. Currently available biomarkers, such as procalcitonin (PCT), provide sepsis diagnostic test results in 2-3 hours with commercially available kits, but to various levels of clinical accuracy [6].

A clinically validated glucose-insulin model that is able to model insulin sensitivity $\left(\mathrm{S}_{\mathrm{I}}\right)$ in real-time has been used to develop blood glucose protocols for critically ill patients [7, 8]. An integral-based parameter identification method has been used to fit the data [9]. The model-based $S_{I}$ has been observed to indicate the severity of illness and metabolic status, as well as being validated against euglycaemic clamp data [10]. Insulin sensitivity has also been previously documented as decreasing with worsening condition [11], and increasing with improvement $[12,13]$.

This study aims to evaluate the relationship of modelled insulin sensitivity $[7,14]$ and patient condition. In particular, this study examines using the modelled insulin sensitivity as a marker for real-time diagnosis and differentiation of Systemic Inflammatory Response Syndrome (SIRS) and sepsis in a cohort of adult ICU patients. It extends the work of Blakemore et al. [15] by increasing discrimination and utilizing additional clinical measurements.

\section{METHODS}

\subsection{Physiological glucose-insulin model}

The physiological glucose-insulin model for clinically ill patients has one compartment for plasma glucose, two compartments for insulin kinetics, and a two-compartment dextrose absorption model.

$$
\begin{aligned}
& \dot{G}=-p_{G} G-S_{I} G \frac{Q}{1+\alpha_{G} Q}+\frac{P(t)+E G P_{b}-C N S}{V_{G}} \\
& \dot{Q}=-k Q+k I \\
& \dot{I}=-\frac{n I}{1+\alpha_{I} I}+\frac{u_{e x}(t)}{V_{I}}+e^{-k_{I} u_{e x}(t)} I_{B} \\
& \dot{P} 1=-d_{1} P 1+D(t)
\end{aligned}
$$


$\dot{P} 2=-\min \left(d_{2} P 2, P_{\max }\right)+d_{1} P(1)$

$P(t)=\min \left(d_{2} P 2, P_{\max }\right)$

Equation (1) represents the plasma glucose compartment where $G$ is the blood glucose level. Equation (2) describes the plasma insulin compartment where $I$ is the plasma insulin level. Equation (3) describes insulin action in interstitial space where $Q$ is the interstitial insulin level. Equations (4)-(6) describe the absorption of dextrose intake $(D(t))$ through stomach (compartment $P 1$ ) and gut (compartment $P 2$ ) and its appearance into plasma $(P(t))$. The transport of glucose from gut to the blood stream is saturable, and the maximum transport rate is $P_{\max }$.

Endogenous glucose production (EGP) for a healthy subject varies throughout a day according to daily activities, food intake and stress response etc. On a pharmacodynamics level, EGP is suppressed with increasing $G$ and $Q$. Time-varying EGP is difficult to measure clinically and cannot be uniquely identified in this study. Therefore this model uses a constant term $E G P_{b}$ in Equation (1) to represent the basal endogenous glucose production for a patient under no presence of glucose or insulin. Insulin independent glucose removal (excluding central nervous system uptake CNS) and the suppression of $E G P$ from $E G P_{b}$ with respect to $G$ are represented with $p_{G}$. In contrast, insulin mediated glucose removal and the suppression of EGP from $E G P_{b}$ due to GLUT4 (which action is associated with the compounding effect of receptor-binding insulin and blood glucose) is represented with $S_{I}$. Insulin sensitivity $\left(S_{I}\right)$ is time varying and reflects evolving patient condition.

In Equation (3), the exogenous insulin administration into plasma insulin is $u_{e x}(t)$. Plasma insulin decay rate is $n$ and $V_{I}$ is the plasma insulin distribution volume. Endogenous insulin production is expressed by $e^{-k_{I} u_{e x}(t)} I_{B}$, where $I_{B}$ is the basal insulin production when no exogenous insulin is present. Endogenous insulin production is suppressed exponentially with exogenous insulin. Table 1 is a nomenclature for all the parameters, symbols and abbreviation used in this article.

This model is an improvement from the model used in [15]. This model has an additional CNS for glucose uptake as this uptake is found to be very consistent inter- and intraindividuals. Dextrose absorption model describes by Equations (4)-(6) is more physiological and universal compare to the simple feed model in [15]. The simple feed model in [15] requires the enteral feeding be relatively constant (not changing more frequently than every 2 hours), whereas the dextrose absorption model presented here is generic and applicable even for modelling meal intake. Finally, this model has an endogenous insulin term $e^{-k_{I} u_{e x}(t)} I_{B}$ to ensure the entire differential equations model in Equations (1)-(6) is not ill-conditioned when there is no exogenous insulin given over a significant period of time. 
Table 1. Nomenclature

\begin{tabular}{|c|c|c|}
\hline$\alpha_{G}$ & $\begin{array}{l}\text { Saturation parameter for insulin mediated } \\
\text { glucose removal }\end{array}$ & $(\mathrm{L} / \mathrm{mU})$ \\
\hline$\alpha_{I}$ & $\begin{array}{l}\text { Saturation parameter for insulin clearance } \\
\text { from plasma }\end{array}$ & $(\mathrm{L} / \mathrm{mU})$ \\
\hline $\mathrm{CDF}$ & Cumulative distribution function & \\
\hline CNS & Central nervous system glucose uptake & (mmol/min) \\
\hline$D(t)$ & Dextrose intake & $(\mathrm{mmol} / \mathrm{min})$ \\
\hline$d_{1}$ & Glucose absorption rate from stomach & $\left(\min ^{-1}\right)$ \\
\hline$d_{2}$ & Glucose absorption rate from gut & $\left(\min ^{-1}\right)$ \\
\hline$E G P$ & Endogenous glucose production & \\
\hline$E G P_{b}$ & Basal endogenous glucose production rate & $(\mathrm{mmol} / \mathrm{min})$ \\
\hline FN & False negative & \\
\hline FP & False positive & \\
\hline$G$ & Blood glucose level & $(\mathrm{mmol} / \mathrm{L})$ \\
\hline GLUT & Glucose transporters & \\
\hline ICU & Intensive Care Unit & \\
\hline$I$ & Plasma insulin level & $(\mathrm{mU} / \mathrm{L})$ \\
\hline$I_{B}$ & Basal endogenous insulin production rate & $(\mathrm{mU} / \mathrm{min} / \mathrm{L})$ \\
\hline$k$ & Interstitial insulin transport rate & $\left(\min ^{-1}\right)$ \\
\hline$k_{1}$ & $\begin{array}{l}\text { Exponential suppression of endogenous } \\
\text { insulin production constant }\end{array}$ & \\
\hline$n$ & Plasma insulin decay rate & $\left(\min ^{-1}\right)$ \\
\hline NPV & Negative predictive value & \\
\hline$P(t)$ & $\begin{array}{l}\text { Glucose appearance in plasma from } \\
\text { dextrose intake }\end{array}$ & $(\mathrm{mmol} / \mathrm{min})$ \\
\hline$P 1$ & Glucose level in stomach & (mmol) \\
\hline$P 2$ & Glucose level in gut & $(\mathrm{mmol})$ \\
\hline PCT & procalcitonin & \\
\hline PDF & Probability density function & \\
\hline$p_{G}$ & $\begin{array}{l}\text { Insulin independent glucose removal } \\
\text { (excluding central nervous system uptake) } \\
\text { and the suppression of } E G P \text { from } E G P_{b} \\
\text { with respect to } G\end{array}$ & $\left(\min ^{-1}\right)$ \\
\hline$P_{\max }$ & Maximal glucose flux from gut to plasma & $(\mathrm{mmol} / \mathrm{min})$ \\
\hline PPV & Positive predictive value & \\
\hline$Q$ & Interstitial insulin level & $(\mathrm{mU} / \mathrm{L})$ \\
\hline ROC & Receiver operator characteristic & \\
\hline$S_{I}$ & $\begin{array}{l}\text { Insulin mediated glucose removal and the } \\
\text { suppression of } E G P \text { from } E G P_{b} \text { with } \\
\text { respect to } G \text { and } Q\end{array}$ & $(\mathrm{~L} / \mathrm{mU} / \mathrm{min})$ \\
\hline SIRS & Systemic inflammatory response score & \\
\hline SOFA & Sepsis-related organ failure assessment & \\
\hline ss & Sepsis score & \\
\hline $\mathrm{TN}$ & True negative & \\
\hline $\mathrm{TP}$ & True positive & \\
\hline$u_{e x}(t)$ & Exogenous insulin & $(\mathrm{mU} / \mathrm{min})$ \\
\hline$V_{I}$ & Plasma insulin distribution volume & (L) \\
\hline$V_{G}$ & Plasma glucose distribution volume & (L) \\
\hline
\end{tabular}

\subsection{Sepsis score (ss) criteria and analysis}

Sepsis is a systemic inflammatory response syndrome (SIRS) due to infection [16]. In this study, sepsis is defined using the clinical classification score (ss) provided by the ACCP/SCCM guideline definitions [17]. The criteria are defined in Tables 2-4, which include SIRS and the Sepsis-related Organ Failure Assessment (SOFA) score [18]. 
Table 2. SIRS criteria

\begin{tabular}{|c|c|c|}
\hline score & \multicolumn{2}{|c|}{ Criteria } \\
\hline+1 & temperature & $\begin{array}{c}<36^{\circ} \mathrm{C} \\
>38^{\circ} \mathrm{C}\end{array}$ \\
\hline+1 & heart rate & $>90 / \mathrm{min}$ \\
\hline+1 & $\begin{array}{c}\text { respiratory rate } \\
\text { or PaCO }\end{array}$ & $\begin{array}{c}>20 / \mathrm{min} \\
<32 \mathrm{~mm} \mathrm{Hg}\end{array}$ \\
\hline+1 & $\begin{array}{c}\text { white blood } \\
\text { cell count }\end{array}$ & $\begin{array}{c}<4 \times 10^{9} / \mathrm{L} \mathrm{or}>12 \times 10^{9} / \mathrm{L} \mathrm{or} \\
\text { presence of }>10 \% \text { immature } \\
\text { granulocytes }\end{array}$ \\
\hline
\end{tabular}

Table 3. SOFA criteria

\begin{tabular}{|c|c|c|c|}
\hline score & system & \multicolumn{2}{|c|}{ criteria } \\
\hline+1 & cardiovascular & $\begin{array}{c}\mathrm{MAP}^{\mathrm{a}} \text { or } \\
\text { need for } \\
\text { inotropes }\end{array}$ & $<60 \mathrm{~mm} \mathrm{Hg}$ \\
\hline+1 & respiratory & $\mathrm{PaO}_{2} / \mathrm{FiO}_{2}$ & $\begin{array}{c}<250 \mathrm{~mm} \mathrm{Hg} / \mathrm{mm} \\
\mathrm{Hg} \\
<200 \mathrm{~mm} \mathrm{Hg} / \mathrm{mm} \\
\mathrm{Hg} \text { with pneumonia }\end{array}$ \\
\hline+1 & renal & Urine output & $<0.5 \mathrm{ml} / \mathrm{kg} / \mathrm{h}$ \\
\hline+1 & blood & platelets & $\begin{array}{c}<80 \times 10^{9} / \mathrm{L} \mathrm{or} 50 \% \\
\text { drop in 3 days }\end{array}$ \\
\hline${ }^{a}$ Mean arterial pressure
\end{tabular}

Table 4. Sepsis score (ss) criteria

\begin{tabular}{|c|c|c|c|c|c|c|c|}
\hline \multicolumn{2}{|c|}{ sepsis score } & SIRS $>2$ & $\begin{array}{c}\text { Infection } \\
\text { during } \\
\text { stay }\end{array}$ & organ failure $>1$ & fluid resuscitation & $\begin{array}{c}\text { high } \\
\text { inotrope present } \\
\text { inotrope } \\
\text { dose }\end{array}$ \\
\hline 0 & normal & & & & \\
\hline 1 & sepsis & $X$ & $X$ & & & \\
\hline 2 & severe sepsis & $X$ & $X$ & $X$ & $X$ & \\
\hline 3 & septic shock & $X$ & $X$ & $X$ & $X$ & $X$ \\
\hline 4 & refractory septic shock & $X$ & $X$ & $X$ & $X$ & $X$ \\
\hline${ }^{2}$ adrenaline or noradrenaline $>0.2 \mathrm{mg} \mathrm{min}^{-1} \mathrm{~kg}^{-1}$ & & & \\
\hline
\end{tabular}

Clinical data was gathered for $\mathrm{n}=36$ sepsis patients admitted to the medical ICU in Christchurch Hospital (Christchurch, New Zealand). Each patient was on the SPRINT blood glucose control protocol [13], providing 9208 total patient hours of hourly modelled insulin sensitivity. The hourly $\mathrm{S}_{\mathrm{I}}$ was compared to SIRS and ss data. Note that each stay included periods of sepsis and without sepsis (ss $=0)$. These periods are differentiated by positive blood culture and SIRS $\geq 2$, and thus ss $\geq 1$.

Receiver Operator Characteristic (ROC) curves were used to examine the performance of $\mathrm{S}_{\mathrm{I}}$ as a diagnostic marker for sepsis. ROC curves effectively examine the ability of the biomarker to differentiate between populations. Sensitivity, specificity, positive predictive value (PPV), and negative predictive value (NPV) were also evaluated. ROC curves effectively examine the separation between normal and diseased populations in terms of probability density functions (PDF). Variablility of ss score from one hour to the next hour were also examined. 
Therefore, other clinical measurements were evaluated and combined with $\mathrm{S}_{\mathrm{I}}$ to create a biomarker that aims to maximise the PDF separation or discrimination in normal and septic groups. The biomarker was created as an hourly changing function based on hourly $\mathrm{S}_{\mathrm{I}}$ and clinical measurements. A linear recursive least square method was used to maximize the discrimination between populations. Optimal weighing functions were chosen using the Optimization Toolbox in Matlab ${ }^{\mathrm{TM}}$. The specific goal was to provide discrimination for $s s \geq 2$, where prior studies [15] only achieved it for $s s \geq 3$. Achieving this goal for the lower ss $=2$ value will provide a marker for a larger group of patients.

Ideally, cross validation or bootstrapping should be performed on the data to avoid overfitting. However, because the current available data is very limited (36 patients), the same data is used for both developing the biomarker and testing it. In particular, the low prevalence of high sepsis score hours limits the ability to boot strap or cross validate effectively. Equally, it should be noted the work presented in this study is more of a proof-of-concept study to justify a larger, more costly comprehensive validation study, rather than a comprehensive large scale validation. The method used in this work is novel in the field of sepsis diagnosis, to the best of the authors' knowledge. Finally, sepsis patient data used in this study is a subset from a cohort representative of typical intensive care patients [13].

\section{RESULTS AND DISCUSSION}

Table 5 shows the total hours at each sepsis score. The majority of the hours are at $s s<2$.

Table 5. Patient hours by sepsis score (ss)

\begin{tabular}{|c|c|c|c|c|c|}
\hline $\begin{array}{c}\text { sepsis } \\
\text { score (ss) }\end{array}$ & 0 & 1 & 2 & 3 & 4 \\
\hline $\begin{array}{c}\text { patient } \\
\text { hours }\end{array}$ & $\begin{array}{c}4186 \\
(45.1 \%)\end{array}$ & $\begin{array}{c}4861 \\
(52.3 \%)\end{array}$ & $\begin{array}{c}91 \\
(1.0 \%)\end{array}$ & $\begin{array}{c}88 \\
(0.9 \%)\end{array}$ & $\begin{array}{c}60 \\
(0.6 \%)\end{array}$ \\
\hline
\end{tabular}

\subsection{Insulin sensitivity $\left(S_{\mathrm{I}}\right)$ and sepsis score (ss)}

Figure 1 shows a cumulative distribution function (CDF) plot of $S_{\text {I }}$, for each ss group. $S_{\text {I }}$ is generally lower for more severe sepsis. However, the distinction between the septic (ss $\geq 1$ ) and non-septic (ss $=0$ ) groups is not clear. 


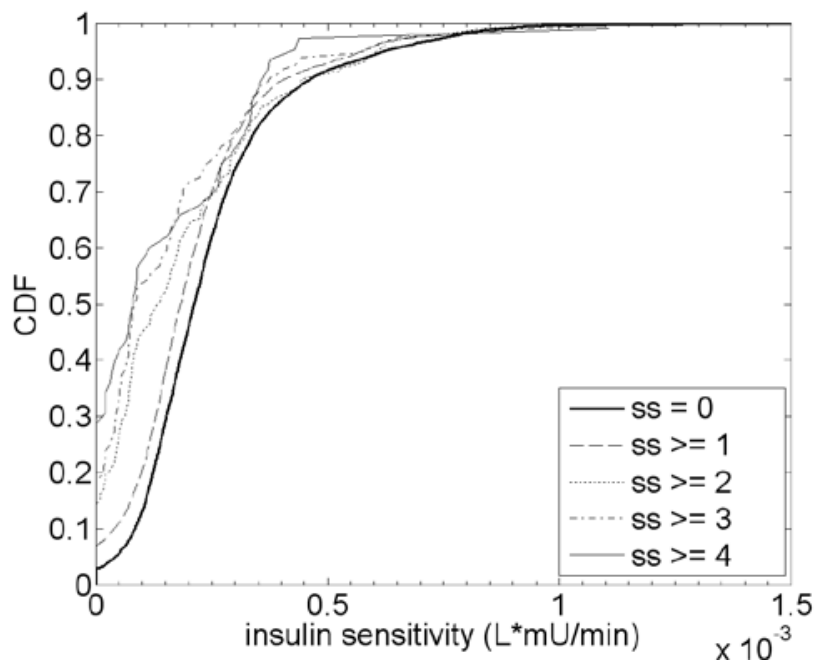

Fig. 1. Cumulative distribution function (CDF) of insulin sensitivity $\left(\mathrm{S}_{\mathrm{I}}\right)$, grouped by sepsis score (ss).

\subsection{Insulin sensitivity $S_{I}$ as a biomarker}

Figure 2 shows the ROC curve for $S_{I}$ as a sepsis biomarker. There is minimal discrimination between no sepsis ( $s s=0$ ) and a sepsis score of $s s=1$. For $\underline{3} s 2$, an $\mathrm{S}_{\mathrm{I}}$ cut-off value of $0.00013 \mathrm{~L} / \mathrm{mU} / \mathrm{min}$ achieves a $50 \%$ sensitivity, $76 \%$ specificity, $4.8 \%$ PPV, and 98.3\% NPV.

Even though $S_{I}$ is generally lower at higher ss values, the distribution of $S_{I}$ for each ss group overlaps too much in Figure 1 with the non septic group. Therefore, using $S_{I}$ level itself is not a completely effective sepsis biomarker.

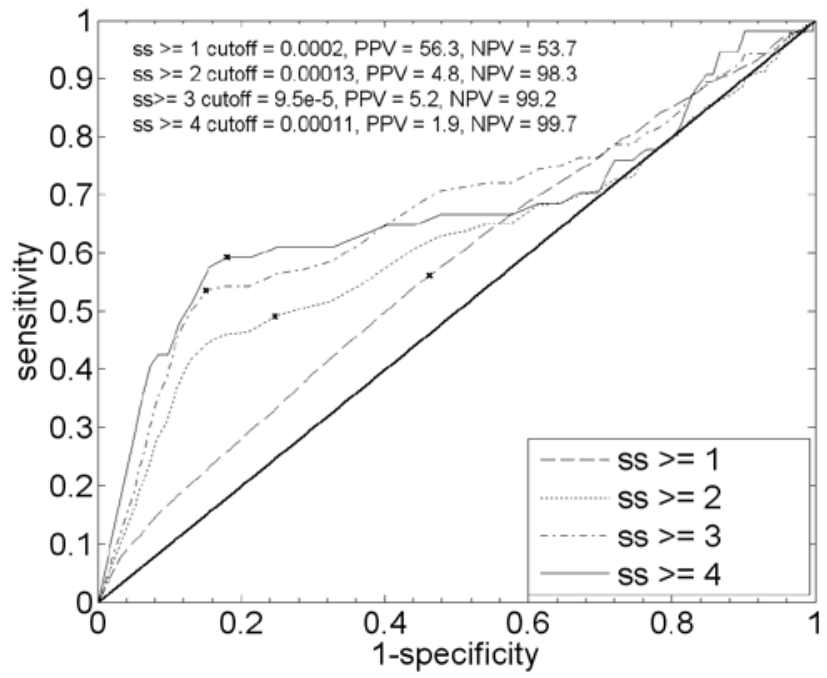

Fig. 2. Receiver operator characteristic (ROC) curve for $\mathrm{S}_{\mathrm{I}}$ as a sepsis biomarker, grouped by sepsis score (ss), with cutoff points (x). 


\subsection{Multivariate clinical biomarker utilising $S_{I}$}

Figure 3 shows the CDF of a biomarker combining $S_{I}$ and other clinical factors, for each ss group. The clinical measurements used in the biomarker include temperature, heart rate, respiratory rate, blood pressure, and their respective rates of change. The multivariate biomarker generally decreases with increasing sepsis severity. The discrimination between sepsis and non-septic groups is improved, as compared to Figure 1 using $\mathrm{S}_{\mathrm{I}}$ only as a diagnostic test.

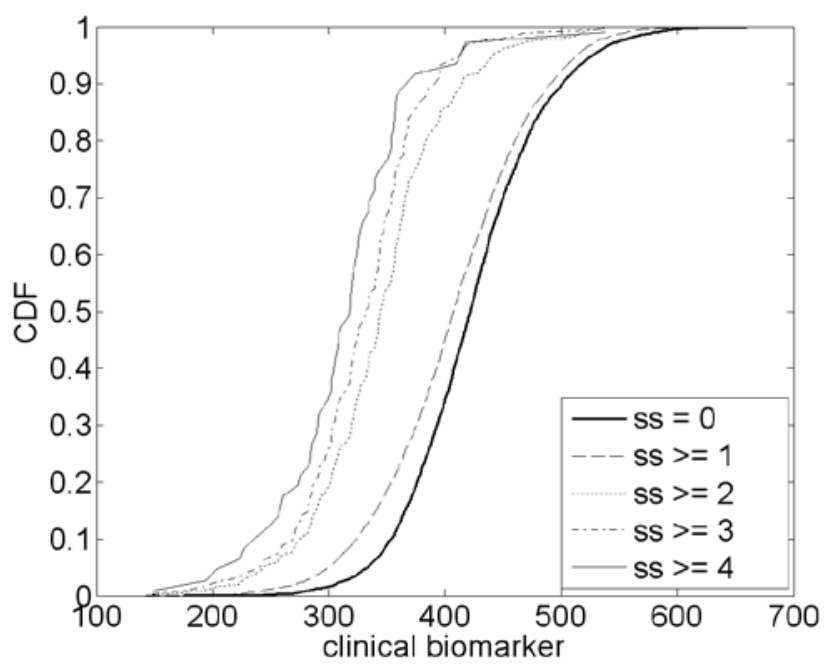

Fig. 3. Cumulative distribution function (CDF) of the clinical biomarker, grouped by sepsis score (ss).

Figure 4 shows the ROC curve for this biomarker. For $s \mathrm{~s} \geq 2$, the biomarker achieves 73\% sensitivity, $80 \%$ specificity, $8.4 \%$ PPV, and $99.2 \%$ NPV. The addition of clinical measurements with $S_{\text {I }}$ significantly improved the diagnostic test performance for sepsis, as compared to using $S_{I}$ alone. In particular, the biomarker provides an effective negative predictive diagnosis for severe sepsis ( $s \mathrm{~s}=2$ ), which was not achieved previously.

It is also clear comparing Figure 2 and Figure 4 that the discrimination between ss $=1$ and $s s \geq 2$ is now wider. Note that $s s=1$ is difficult to discriminate from $s s=0$ and simple, clinical SIRS. Hence, as seen in Figure 2 and Figure 4, its discrimination from ss $=0$ is still marginal. 


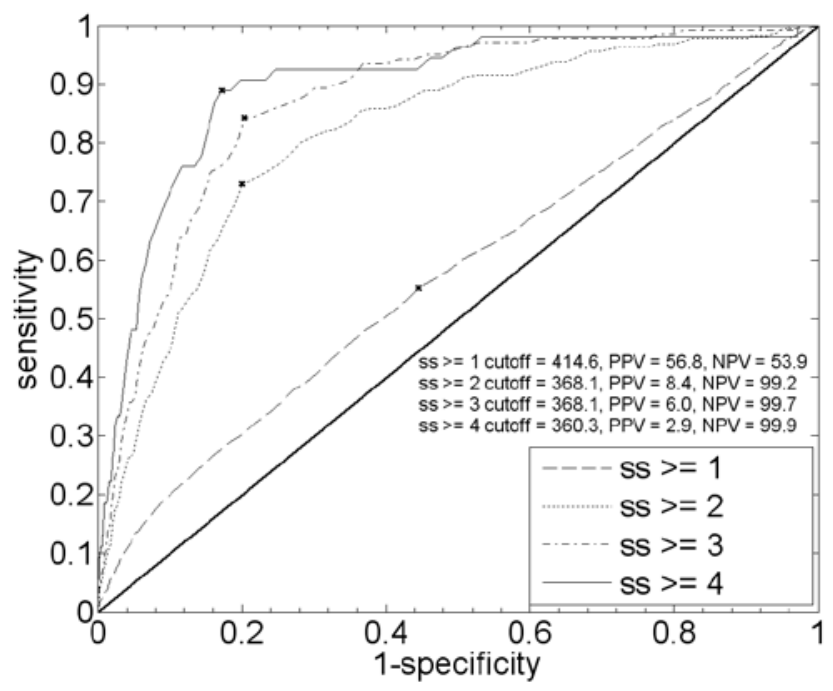

Fig. 4. Receiver operator characteristic (ROC) curve for the multivariate clinical biomarker, grouped by sepsis score (ss), with cutoff points (x).

Table 6 is a contingency table showing the biomarker diagnostic outcome for $8 \mathrm{~s}$ There are 9208 total hours of patient data. This data is classified into four categories using sepsis score and biomarker test outcome: true positive (TP, $\mathrm{n}=165$ ), false positive ( $F P, n=1802)$, false negative $(F N, n=61)$, and true negative $(T N, n=7180)$. There are 8982 hours when ss $<2$ and 226 hours when ss $\geq 2$. Using the biomarker, 7241 hours test negative and 1967 hours test positive.

Table 6. Contingency table

\begin{tabular}{|c|c|c|c|}
\cline { 2 - 3 } \multicolumn{1}{c|}{} & ss $\geq 2$ & ss $<2$ & \multicolumn{1}{c}{} \\
& 226 hours & 8982 hours & \\
\hline test positive & $\mathrm{TP}=165$ & $\mathrm{FP}=1802$ & $\mathrm{PPV}$ \\
1967 hours & $(1.8 \%)$ & $(19.6 \%)$ & $8.4 \%$ \\
\hline test negative & $\mathrm{FN}=61$ & $\mathrm{TN}=7180$ & $\mathrm{NPV}$ \\
7241 hours & $(0.7 \%)$ & $(78 \%)$ & $99.2 \%$ \\
\hline total & sensitivity & specificity & \multicolumn{1}{c}{} \\
9208 hours & $73.0 \%$ & $79.9 \%$ & \multicolumn{1}{c}{} \\
\hline
\end{tabular}

Note that these ratios also indicate the relative incidence and reflect clinical expectations where, by the hour, most sepsis is not necessarily severe. This last point is critical as most sepsis incidence is recorded by patients. By patient, the incidence of severe sepsis is 5$10 \%$ [1], which is reflected in this cohort. However, with rapid, aggressive treatment its incidence by hour is low. This low incidence is problematic in developing such a noninvasive and real-time clinical biomarker.

Figure 5 provides a visual presentation of clinical sepsis score and the biomarker performance. Most of the patient hours are ss $<2$ (TN + FP). Even though the biomarker correctly identifies the severity of sepsis $73 \%$ of the time when $\underline{\underline{s}} \mathbf{2}$ and $80 \%$ of the time when ss $<2$, the PPV stays low because the ratio of TP to test positive is limited by the ratio between $s s \geq 2$ to $s s<2$. On contrast, NPV is inherently high because the ratio of TN makes up the majority of test negative. Again, the mathematics indicate that 
relatively low incidence by hour, as seen in the 8982 hours of ss $<2$ and 4186 hours of ss $=0$, hinders good PPV despite good specificity.

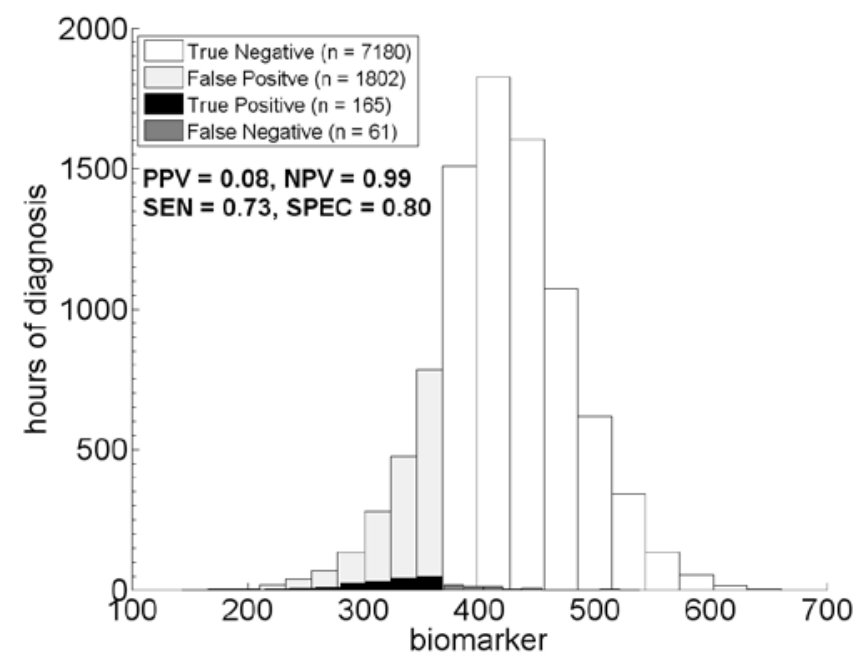

Fig. 5. Histogram of clinical biomarker data, grouped by contingency results.

\subsection{Sepsis time course}

Figure 6 effectively shows the probability of how ss changes from one hour to the next. The horizontal axis is the current hour ss and the vertical axis is the ss for the next hour. The majority of the patient data is when $\mathrm{ss}=0$ and $\mathrm{ss}=1$.

Patients at ss $=1$ tend to stay at ss $=1$, and patients having ss $=0$ tend to stay at ss $=0$. Interestingly, when $s s \geq 2$, the highest probability is moving to $\mathrm{ss}=1$ in the next hour. This set of results shows that if sepsis is detected at $s \underline{s} 2$, current rapid and agg ressive ICU treatments are usually very effective in reducing the severity of the inflammatory responses.

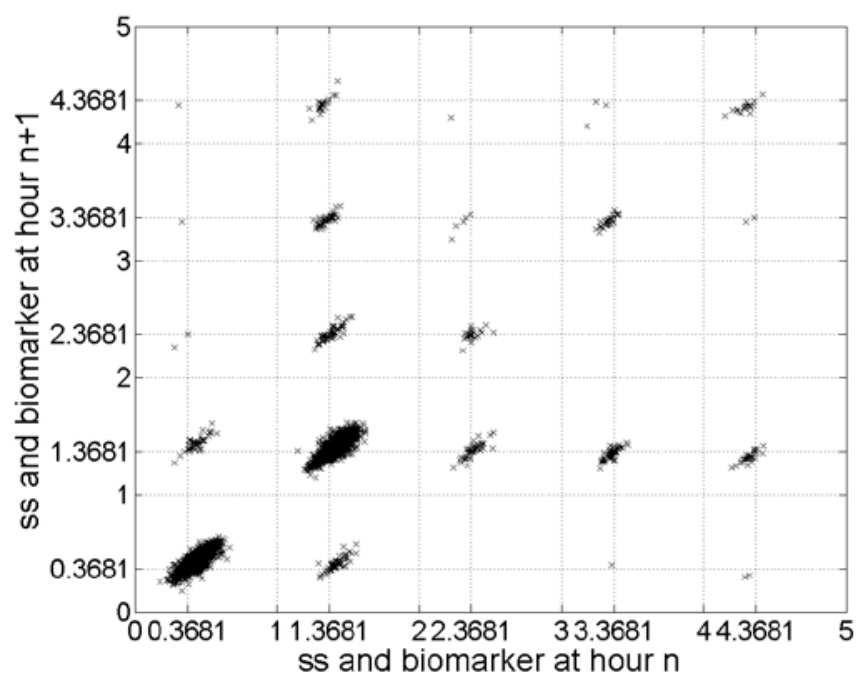


Fig. 6. Scatter plot of clinical biomarker variation from hour to hour with respect to sepsis score (ss) (axis numbers = ss + biomarker/1000).

\subsection{Biomarker comparison}

The clinical informative values a biomarker may provide in sepsis are: detection of infection; diagnosis of SIRS severity and infection progression; and patient treatment guidance, responsiveness, and prognosis. One such investigated biomarker is procalcitonin (PCT), which was first found elevated in sepsis in 1993 [19]. PCT is a precursor of the hormone calcitonin, synthesized by thyroid $\mathrm{C}$ cells, but in sepsis has an extra-tyroidal origin. Upon intravenous injection of endotoxin from E. coli in healthy volunteers, serum PCT becomes detectable after 4 hours, maintaining a plateau through 8 to 24 hours, following an increase of proinflammatory cytokines [20].

PCT can be measured from serum plasma by commercially available immunoluminometric assay kits such as LUMItest PCT (Brahms, Berlin, Germany) and Kryptor PCT (Brahms, Hennigsdorf, Germany). The use of PCT has been approved by the FDA "in conjunction with other laboratory findings and clinical assessments to aid in the risk assessment of critically ill patients on their first day of ICU admission for progression to severe sepsis and septic shock".

The reported diagnostic power of PCT from 25 studies using PCT (2,966 patients) as a diagnostic marker of sepsis, severe sepsis, or septic shock in the adult ICU or after surgery or multiple trauma, compared with nonseptic SIRS has sensitivity ranging from $42 \%$ to $97 \%$ or even $100 \%$, and specificity ranging from $48 \%$ to $100 \%$ [21]. Optimal cutoff values for PCT, determined from ROC curves, ranged from 0.6 to $5 \mathrm{ng} / \mathrm{mL}$.

PCT is generally only assessed once a day and thus cannot provide real-time detection. In contrast, the biomarker presented in this study is a real-time marker. In addition, all of these studies had some form of extensive clinical pre-screening for sepsis and/or SIRS, biasing the sensitivity or specificity. The overall results reported are no better, and often worse, than those reported here.

Traditionally, diagnostic test results are based on a cut-off point. This method effectively treats all patients as a single, generic person with one clear cut-off point that distinguishes between normal and abnormal states. However, recent medical treatments have been moving towards patient customisation - tailoring treatments to patient needs [22]. Although this study has so far provided a sepsis biomarker that has a cut-off value which can differentiate ss $\geq 2$ from ss $<2$ most of the time, this biomarker is also a time-varying value. As with most clinical measurements, this biomarker would have both inter- and intra-patient statistical distribution.

\subsection{Future work}

It is therefore of interest to further investigate how this biomarker changes through time for patients, and how much variability there is between patients. A means of normalizing patients and likely changes using stochastic models would improve biomarker performance. An observational study and follow-up of a biomarker for sepsis diagnosis in the ICU would provide the data needed for that analysis. Finally, the statistical model of a 
biomarker, like the one developed in this paper, may be more useful clinically by providing a probability analysis of disease progression in real-time.

\section{CONCLUSIONS}

Insulin sensitivity as a sepsis biomarker for diagnosis of severe sepsis achieves a $50 \%$ sensitivity, 76\% specificity, 4.8\% PPV, and $98.3 \% \mathrm{NPV}$ at an $\mathrm{S}_{\mathrm{I}}$ cut-off value of 0.00013 $\mathrm{L} / \mathrm{mU} / \mathrm{min}$. A discriminating threshold was not found between the ss $=0$ and $\mathrm{ss}=1$ cohorts due to a high degree of population overlap. A clinical biomarker combining $\mathrm{S}_{\mathrm{I}}$, temperature, heart rate, respiratory rate, blood pressure, and the respective rate of change achieves 73\% sensitivity, 80\% specificity, 8.4\% PPV, and 99.2\% NPV, and thus can act as an effective negative predictive diagnosis for severe sepsis.

PPV performance is poor because the ratio of true positive to test positive is limited by the ratio of hours where ss $\geq 2$ to $s s<2$. The majority of patient hours had ss $<2$. However, the multivariate clinical biomarker may provide patient-specific diagnosis, and effectively show the probability of sepsis time course from hour to hour. Real-time results may aid in the treatment and management of sepsis in the ICU. Future work includes an observational study and follow-up of a biomarker for sepsis diagnosis in the ICU.

\section{REFERENCES}

[1] D. Angus, W. Linde-Zwirble, J. Lidicker, G. Clermont, J. Carcillo, and M. Pinsky, Epidemiology of severe sepsis in the United States: Analysis of incidence, outcome, and associated costs of care, Critical Care Medicine, (2001) 29(7): p. 1303.

[2] R. Dellinger, M. Levy, J. Carlet, J. Bion, M. Parker, R. Jaeschke, K. Reinhart, D. Angus, C. Brun-Buisson, and R. Beale, Surviving Sepsis Campaign: International guidelines for management of severe sepsis and septic shock: 2008, Critical Care Medicine, (2008) 36(1): p. 296.

[3] G. Martin, D. Mannino, S. Eaton, and M. Moss, The Epidemiology of Sepsis in the United States from 1979 through 2000, New England Journal of Medicine, (2003) 348(16): p. 1546-1554.

[4] E. Rivers, B. Nguyen, S. Havstad, J. Ressler, A. Muzzin, B. Knoblich, E. Peterson, and M. Tomlanovich, Early Goal-Directed Therapy in the Treatment of Severe Sepsis and Septic Shock, New England Journal of Medicine, (2001) 345(19): p. 1368-1377.

[5] G. Van den Berghe, P. Wouters, F. Weekers, C. Verwaest, F. Bruyninckx, M. Schetz, D. Vlasselaers, P. Ferdinande, P. Lauwers, and R. Bouillon, Intensive Insulin Therapy in Critically Ill Patients, New England Journal of Medicine, (2001) 345(19): p. 1359-1367.

[6] S. Carrigan, G. Scott, and M. Tabrizian, Toward Resolving the Challenges of Sepsis Diagnosis, Clinical Chemistry, (2004) 50(8): p. 1301-1314.

[7] T. Lonergan, A. Compte, M. Willacy, J. Chase, G. Shaw, X. Wong, T. Lotz, J. Lin, and C. Hann, A Simple Insulin-Nutrition Protocol for Tight Glycemic Control 
in Critical Illness: Development and Protocol Comparison, Diabetes Technology \& Therapeutics, (2006) 8(2): p. 191-206.

[8] J.G. Chase, G.M. Shaw, T. Lotz, A. LeCompte, J. Wong, J. Lin, T. Lonergan, M. Willacy, and C.E. Hann, Model-based insulin and nutrition administration for tight glycaemic control in critical care, Curr Drug Deliv, (2007) 4(4): p. 283-96.

[9] C.E. Hann, J.G. Chase, J. Lin, T. Lotz, C.V. Doran, and G.M. Shaw, Integralbased parameter identification for long-term dynamic verification of a glucoseinsulin system model, Comput Methods Programs Biomed, (2005) 77(3): p. 259270.

[10] T.F. Lotz, J.G. Chase, K.A. McAuley, D.S. Lee, J. Lin, C.E. Hann, and J.I. Mann, Transient and steady-state euglycemic clamp validation of a model for glycemic control and insulin sensitivity testing, Diabetes Technol Ther, (2006) 8(3): p. 33846.

[11] C. Chambrier, M. Laville, K. Berrada, M. Odeon, P. Bouletreau, and M. Beylot, Insulin sensitivity of glucose and fat metabolism in severe sepsis, CLINICAL SCIENCE, (2000) 99(4): p. 321-328.

[12] L. Langouche, S. Vander Perre, P.J. Wouters, A. D'Hoore, T.K. Hansen, and G. Van den Berghe, Effect of Intensive Insulin Therapy on Insulin Sensitivity in the Critically Ill, Journal of Clinical Endocrinology \& Metabolism, (2007) 92(10): p. 3890.

[13] J.G. Chase, G. Shaw, A. Le Compte, T. Lonergan, M. Willacy, X.-W. Wong, J. Lin, T. Lotz, D. Lee, and C. Hann, Implementation and evaluation of the SPRINT protocol for tight glycaemic control in critically ill patients: a clinical practice change, Critical Care, (2008) 12(2): p. R49.

[14] J. Lin, D. Lee, J. Chase, C. Hann, T. Lotz, and X. Wong, Stochastic Modelling of Insulin Sensitivity Variability in Critical Care, Biomedical Signal Processing \& Control, (2006) 1: p. 229-242.

[15] A. Blakemore, S. Wang, A.J. Le Compte, G.M. Shaw, X.W. Wong, J. Lin, T. Lotz, C.E. Hann, and J.G. Chase, Model-Based Insulin Sensitivity as a Sepsis Diagnostic in Critical Care, Journal of Diabetes Science and Technology, (2008) 2(3): p. 468-77.

[16] R. Bone, Definitions for sepsis and organ failure and guidelines for the use of innovative therapies in sepsis. The ACCP/SCCM Consensus Conference Committee. American College of Chest Physicians/Society of Critical Care Medicine, Chest, (1992) 101(6): p. 1644-1655.

[17] M. Levy, M. Fink, J. Marshall, E. Abraham, D. Angus, D. Cook, J. Cohen, S. Opal, J. Vincent, and G. Ramsay, 2001 SCCM/ESICM/ACCP/ATS/SIS International Sepsis Definitions Conference, Critical Care Medicine, (2003) 31(4): p. 1250.

[18] J. Vincent, R. Moreno, J. Takala, S. Willatts, A. De Mendonca, H. Bruining, C. Reinhart, P. Suter, and L. Thijs, The SOFA (Sepsis-related Organ Failure Assessment) score to describe organ dysfunction/failure. On behalf of the Working Group on Sepsis-Related Problems of the European Society of Intensive Care Medicine, Intensive Care Med, (1996) 22(7): p. 707-10. 
[19] M. Assicot, D. Gendrel, H. Carsin, J. Raymond, J. Guilbaud, and C. Bohuon, High serum procalcitonin concentrations in patients with sepsis and infection, Lancet, (1993) 341(8844): p. 515-8.

[20] P. Dandona, D. Nix, M. Wilson, A. Aljada, J. Love, M. Assicot, and C. Bohuon, Procalcitonin increase after endotoxin injection in normal subjects, J Clin Endocrinol Metab, (1994) 79(6): p. 1605-1608.

[21] B. Uzzan, R. Cohen, P. Nicolas, M. Cucherat, and G.-Y. Perret, Procalcitonin as a diagnostic test for sepsis in critically ill adults and after surgery or trauma: a systematic review and meta-analysis, Critical Care Medicine, (2006) 34(7): p. 1996-2003.

[22] J. Chase, C. Hann, G. Shaw, X. Wong, J. Lin, T. Lotz, A. Le Compte, and T. Lonergan, An overview of glycemic control in critical care-relating performance and clinical results, Journal of Diabetes Science and Technology, (2007) 1: p. 8291. 